\title{
Botulinum-A Toxin Injections Into the Detrusor Muscle Decrease Nerve Growth Factor Bladder Tissue Levels in Patients With Neurogenic Detrusor Overactivity
}

\author{
Antonella Giannantoni,* Savino M. Di Stasi, Vincenza Nardicchi, Alessandro Zucchi, \\ Lara Macchioni, Vittorio Bini, Gianfrancesco Goracci and Massimo Porena \\ From the Paediatric (VB) and Biochemistry (VN, LM, GG) Sections, Department of Internal Medicine and Department of Urology \\ (AG, AZ, MP), University of Perugia, Perugia and Department of Urology, "Tor Vergata" University of Rome (SMDS), Rome, Italy
}

\begin{abstract}
Purpose: We investigated the effects of BTX-A on visceral afferent nerve transmission by measuring bladder tissue NGF levels in patients with neurogenic detrusor overactivity before and after intravesical treatment with BTX-A. We also compared the bladder tissue NGF content with clinical and urodynamic data.

Materials and Methods: A total of 23 patients underwent clinical evaluation and urodynamics with detection of the UDC threshold, maximum pressure and maximum cystometric capacity before, and at the 1 and 3-month followups. Endoscopic bladder wall biopsies were also obtained at the same time points. NGF levels were measured in tissue homogenate by enzyme-linked immunosorbent assay (Promega, Madison, Wisconsin).

Results: At 1 and 3 months mean catheterization and incontinent episodes were significantly decreased ( $p<0.05$ and $<0.001$, respectively). On urodynamics we detected a significant increase in the UDC threshold and maximum cystometric capacity, and a significant decrease in UDC maximum pressure at the 1 and 3-month followups compared to baseline (each $\mathrm{p}<0.001$ ). At the same time points we detected a significant decrease in NGF bladder tissue content $($ each $\mathrm{p}<0.02)$.

Conclusions: BTX-A intravesical treatment induces a state of NGF deprivation in bladder tissue that persists at least up to 3 months. As caused by BTX-A, the decrease in acetylcholine release at the presynaptic level may induce a decrease in detrusor contractility and in NGF production by the detrusor muscle. Alternatively BTX-A can decrease the bladder level of neurotransmitters that normally modulate NGF production and release.
\end{abstract}

Key Words: spinal cord injuries, bladder, botulinum toxin type A, nerve growth factor

$\mathrm{B}$ TX-A is an extremely potent neurotoxin that acts by blocking calcium dependent $\mathrm{ACh}$ release at the peripheral neuromuscular junction. ${ }^{1,2}$ Although BTX-A is known to induce transient chemodenervation of striated and smooth muscles, there is experimental and clinical evidence of a certain effect on afferent C-fibers, which should explain the antinociceptive effect on bladder tissue in rat chemical cystitis models $^{2}$ and in patients with interstitial cystitis. ${ }^{1}$

An observation is that the neurotoxin inhibits the release of neurotransmitters from the terminals of primary nociceptive afferents by its proteolytic light chain, which hydrolyzes key components of the SNARE (SNAP receptor) complex required for synaptic vesicle docking, fusion and neurotransmitter release. ${ }^{3}$ Recently the ability of a catalytically active derivative of BTX-A to block secretion from vesicle populations in dorsal root ganglia neuronal cultures was noted for the release of substance $\mathrm{P}$ and glutamate, which are involved in afferent nociceptive transmission to the central nervous system. ${ }^{3}$

Experimental studies indicate that detrusor overactivity after SCI is induced at least in part by phenotypic changes in

Submitted for publication July 22, 2005.

Study received Ethics Committee approval.

* Correspondence: Department of Urology, University of Perugia, Policlinico Monteluce, Via Brunamonti 51, Perugia, Italy (telephone: 0039075 5783979; FAX: 0039075 5726123; e-mail: agianton@tin.it). unmyelinated C-fiber bladder afferent pathways. ${ }^{4}$ The hyperexcitability of $\mathrm{C}$-fiber bladder afferents may be mediated at least in part by an increase in NGF, which is produced in target organ tissues and transported retrograde back to the neuronal cell bodies. ${ }^{5}$

Taken together these findings suggest that BTX-A may inhibit detrusor overactivity by acting on the efferent and afferent arms of the micturition reflex. Thus, in addition to the inhibition of $\mathrm{ACh}$ release at the presynaptic level, there may be another mechanism of action of BTX-A, eventually involving NGF production and/or release.

We measured bladder tissue NGF levels in patients with neurogenic detrusor overactivity before and after BTX-A injections into the detrusor muscle. We compared the bladder tissue NGF content with clinical and urodynamic data.

\section{MATERIALS AND METHODS}

\section{Patient Characteristics}

This study enrolled 13 males and 10 females with chronic SCI and detrusor overactivity unresponsive to conventional anticholinergic therapy. Mean age \pm SD was $37.6 \pm 11.4$ years and the mean disease duration was $40.5 \pm 17.8$ months. Neurological clinical evaluation was done according to the American Spinal Injury Association classification ${ }^{6}$ with type A impairment in 15 patients and type B impairment in 8 . A total of 18 patients had a lesion at the thoracic 


\begin{tabular}{|c|c|c|c|c|}
\hline \multicolumn{5}{|c|}{ Clinical, urodynamic and NGF results in SCI patients before and after BTX-A treatment } \\
\hline & $\begin{array}{c}\text { Mean Before } \\
\text { Treatment } \pm \text { SD }\end{array}$ & Mean $1 \mathrm{Mo} \pm \mathrm{SD}$ & Mean 3 Mos \pm SD & $\mathrm{p}$ Value \\
\hline Threshold (ml) & $165 \pm 92.2$ & $420.4 \pm 105.6$ & $445.5 \pm 52$ & $<0.001$ \\
\hline Max pressure $\left(\mathrm{cm} \mathrm{H}_{2} \mathrm{O}\right)$ & $62.3 \pm 21.8$ & $17 \pm 20.7$ & $24.6 \pm 32.5$ & $<0.001$ \\
\hline
\end{tabular}

level, while the remaining patients showed cervical injury. The study was approved by the Ethics Committee. All patients were informed about the scientific nature of the study and provided written consent.

\section{Preliminary Assessment}

Standard evaluation included history, physical examination, serum chemistry studies, urinalyses, urine culture and imaging assessment of the urinary tract by ultrasound, excretory urography and cystourethrography. Baseline urodynamics done according to International Continence Society Standards ${ }^{7}$ were performed 1 month before commencing the study. Maximum UDC pressure, the lowest volume (threshold) at which contractions occurred and maximum bladder capacity were recorded. Inclusion criteria were unacceptable clinical (patient driven) and urodynamic suppression of detrusor activity (our assessment) by oral and intravesical oxybutynin, intolerable anticholinergic side effects, normal renal function and a normal upper urinary tract. Patients with myasthenia gravis and other diseases affecting cholinergic action, pregnancy and concomitant use of aminoglycosides were excluded from study.

\section{Study Plan}

All 23 patients fulfilling all criteria were selected and included in the study. They underwent cystoscopy with bladder wall biopsy specimens obtained from the posterolateral wall of the bladder with cold cup resection. We performed deep bladder wall biopsy, likely including urothelium and smooth muscle. No biopsies were taken from the bladder neck or the trigone area.

\section{BTX-A Injections into the Detrusor Muscle}

After undergoing cystoscopy with biopsy specimens patients were injected with $300 \mathrm{U}$ commercially available BTX-A diluted in $30 \mathrm{ml} 0.9 \% \mathrm{NaCl}$ into the detrusor muscle at 30 sites, sparing the trigone, under cystoscopic guidance. In 8 patients the procedure was performed under spinal anesthesia on an inpatient basis. The remaining procedures were performed with the patient under sedation only.

Urinary symptoms were assessed with a voiding diary maintained by patients for 30 days before commencing the study and for 3 months throughout followup. Patients recorded the number of daily catheterizations and episodes of incontinence.

Urodynamic studies and cystoscopies with biopsy specimens were repeated 1 and 3 months later. Local and/or systemic side effects were noted during and after treatment.

\section{NGF Assay}

Bladder specimens ( 1 to $2 \mathrm{mg}$ ) were rapidly frozen and stored at $-80 \mathrm{C}$. Samples were homogenized using a frosted glass potter in $400 \mu$ l lysis buffer, composed of $137 \mathrm{mM}$ $\mathrm{NaCl}, 20 \mathrm{mM}$ tris-HCl (pH 8.0) 1\% NP40, 10\% glycerol, 1 $\mathrm{mM}$ phenylmethylsulfonyl fluoride, $10 \mu \mathrm{g} / \mathrm{ml}$ aprotinin, 1 $\mu \mathrm{g} / \mathrm{ml}$ leupeptin and $0.5 \mathrm{mM}$ sodium vanadate (Promega). The homogenate was centrifuged at $2,000 \times$ gravity for 15 minutes at $4 \mathrm{C}$. NGF levels were measured in the soluble fraction after determining total protein concentration by the Bradford assay with $22 \mu$ l of each sample diluted 1:2 with lysis buffer and then with Dulbecco's phosphate buffered saline buffer, diluted 1:5. Samples were acidified, maintained at $\mathrm{pH} 2.0$ to 3.0 for 20 minutes and then neutralized with $1 \mathrm{~N} \mathrm{NaOH}$. Acidification increased assay sensitivity, likely due to the proteolysis of $7 \mathrm{~S}$ to the $2.5 \mathrm{~S}$ form or to its dissociation from binding protein. ${ }^{8}$

Because the NGF Emax® ImmunoAssay System contains an anti-rat conjugate that cross-reacts with human IgG, the standard procedure was modified by immunoprecipitating IgGs with rheumatoid factor (IgM) before enzyme-linked immunosorbent assay. For this reason neutralized extracts were treated with the addition of rheumatoid factor (IgM, 1:10 volume by volume) for 10 minutes at $4 \mathrm{C}$. They were then centrifuged at $2,000 \times$ gravity for 10 minutes. NGF levels were measured in $100 \mu \mathrm{l}$ supernatant by enzymelinked immunosorbent assay. Sensitivity was $15.6 \mathrm{pg} / \mathrm{ml}$ NGF. Results are expressed in pg NGF/ $\mu$ g protein.

\section{Data Analysis}

Data analysis was performed using the nonparametric Friedman test, followed by the Wilcoxon signed ranks test for paired data with the Bonferroni correction for multiple comparisons and the Spearman rho coefficient for nonparametric bivariate correlations. Statistical significance was considered at $\mathrm{p}<0.05$. All calculations were performed with SPSS, release 13.0 (SPSS, Chicago, Illinois).

\section{RESULTS}

\section{Pretreatment Assessment}

All patients performed clean intermittent catheterization and received oral anticholinergics (oxybutynin $5 \mathrm{mg} 3$ times daily). All had incontinence episodes and used pads or external collecting devices.

Urodynamics revealed detrusor overactivity with detrusor-sphincter dyssynergia in 14 patients, and decreased bladder capacity and urinary incontinence in all. All urinary tract infections were treated. All patients were free from infection at the beginning of the study and during followup. 


\section{Followup}

The table lists clinical results. At the 1 and 3-month followups mean catheterization and incontinent episodes were significantly decreased compared to baseline $(\mathrm{p}<0.05$ and $<0.001$, respectively). A total of 18 patients achieved complete urinary continence, which was maintained during followup.

On urodynamics we detected a significant increase in the UDC threshold and in maximum cystometric capacity at the 1 and 3-month followups (each $\mathrm{p}<0.001$, see table). Furthermore, at the same time points we observed a significant decrease in UDC maximum pressure compared to baseline $(p<0.001)$. No significant differences were found in the UDC threshold and maximum pressure or in maximum bladder capacity between the 1 and 3-month followups.

We did not observe any local or systemic side effects during or after treatment. Despite clinical and urodynamic benefits all patients continued to receive anticholinergics (oxybutynin $5 \mathrm{mg} 3$ times daily) during the entire observation to maintain the same conditions as before treatment.

The table shows NGF results. We detected a significant decrease in bladder tissue NGF content at the 1 and 3 -month followups compared to baseline (each $\mathrm{p}<0.02$ ). We did not observe any significant relationship between bladder tissue NGF levels and patient disease duration or between NGF and the level of SCI.

At the 1 and 3-month followups we detected a positive trend in the relationship between high NGF levels and the frequency of daily incontinent episodes. Furthermore, there was a negative trend in the relationship between high NGF levels, and a low UDC threshold and low bladder capacity. At the 1 and 3-month followups a positive trend was observed in the relationship between high NGF levels and low UDC maximum pressure. At 1 month the Spearman correlation coefficient showed a significant relationship between the $\Delta$ NGF and $\Delta$ UDC threshold $(\mathrm{r}=-0.520, \mathrm{p}<0.05)$ and between $\Delta$ NGF and $\Delta$ UDC maximum pressure $(\mathrm{r}=0.545, \mathrm{p}<0.05)$.

In 6 patients the bladder tissue NGF content was measured again at 6 months of followup and the mean value was $94.06 \pm 42.1 \mathrm{ng} / \mathrm{mg}$, thus, indicating an initial increase in the neurotransmitter. This observation must be confirmed in all patients.

\section{DISCUSSION}

Experimental studies have shown a peripheral antinociceptive effect of BTX-A independent of its actions at the neuromuscular junction of striated and smooth muscle. ${ }^{1}$ In vivo the role of nociceptive afferents is to sense noxious stimuli at the periphery and transmit them to the central nervous system, where they are perceived as pain. Transmission of this signal depends on the release of a number of transmitters, including glutamate, substance $\mathrm{P}$ and CGRP, from synaptic vesicles. ${ }^{3}$ Duggan et al noted that a conjugate obtained by the coupling of a derivative of BTX-A with a lectin shows in vitro selectivity for nociceptive afferents, resulting in the inhibition of neurotransmitters from synaptic vesicles of dorsal root ganglia neuronal cultures. ${ }^{3}$ In addition, it was recently reported that intravesical BTX-A significantly decreases afferent nerve mediated contractions to electrical and chemical stimulation in treated rats compared to those in saline treated animals. ${ }^{9}$ Thus, there may be a possible neurotoxin mediated block of the release of neurotransmitters from bladder afferent terminals.
NGF has a key role in the survival of sensory neurons not only during development, but also throughout adulthood for maintaining the normal properties of $\mathrm{C}$ afferent fibers. ${ }^{6}$ The intravesical administration of exogenous NGF in animals may facilitate afferent firing and cause bladder hyperactivity, which is blocked by anti-nerve growth factor. ${ }^{6}$ It has been documented that after spinal cord injury $\mathrm{C}$ afferent fibers initiate a usually inactive, involuntary spinal reflex and NGF may itself decrease the C-fiber threshold. ${ }^{10}$ Unfortunately data on NGF bladder tissue determination in humans are limited. ${ }^{6,11-13}$ To our knowledge there is no consistent information about bladder NGF in patients with SCI before and after intravesical treatment with BTX-A.

Taken together this information led us to speculate that there may be certain relationships between the mechanisms of action of the neurotoxin, and the production and effects of NGF at the level of bladder afferent nerve transmission. The results of this study demonstrate that BTX-A injected into the bladder wall of patients with SCI induced significant amelioration of clinical conditions and detrusor overactivity. Simultaneously bladder tissue NGF levels significantly decreased 1 and 3 months after treatment.

An explanation may be that the decreased $\mathrm{ACh}$ release at the presynaptic level caused by BTX-A induces a decrease in detrusor contractility and NGF production. In this regard it has previously been observed that cyclic stretch of cultured bladder smooth muscle elicits rapidly increased expression of NGF mRNA and high NGF levels in the culture medium. ${ }^{14}$

Another possibility is that the decrease in NGF after BTX-A can be due to decreased neurogenic inflammation, a phenomenon probably produced by an axon reflex involving antidromic propagation of impulses through the terminal branches of sensory nerve axons. ${ }^{15}$ These axons release inflammatory mediators, such as substance $\mathrm{P}$, neurokinin A and CGRP, into peripheral tissues, which exert direct vasoactive effects (vasodilatation and increased vasopermeability) with consequent tissue edema. The block of secretion from vesicle populations in dorsal root ganglia neuronal cultures of substance $\mathrm{P}$ and glutamate, as induced by BTX$\mathrm{A},{ }^{3}$ can decrease the effects of neurogenic inflammation. Furthermore, during followup patients did not show any urinary tract infection and the majority did not report any infection at a mean followup of 6 months.

A more intriguing hypothesis comes from the observation that BTX-A inhibits not only the release of $\mathrm{ACh}$ and noradrenaline, ${ }^{16}$ but also that of ATP, substance P and CGRP, from the detrusor muscle and urothelium. ${ }^{17} \mathrm{ACh}$, noradrenaline and ATP have been demonstrated to decrease NGF production at the level of the detrusor muscle and urothelium. ${ }^{11}$

One may argue that BTX-A is known to induce chemical denervation of the detrusor muscle, which could increase bladder tissue NGF levels. Indeed, after BTX-A intravesical treatment we found a decrease in neurotransmitter content. This may have been the result of 2 actions, namely denervation on one hand and block in the release of several neurotransmitters (ACh, adrenaline and ATP) on the other hand.

In fact, the mechanisms underlying these results may be more complex and to our knowledge the relationships between BTX-A and NGF have yet to be clarified. NGF receptor binding, NGF internalization and NGF transport have been identified as a potential route of delivery for several 
molecules, including BTX-A, ${ }^{18}$ but the meaning of this activity is still unclear. Furthermore, NGF is produced not only by the bladder, but also by the injured spinal cord and it is then transported to the afferents as a neurotransmitter. ${ }^{19}$ Thus, it is possible that BTX-A can block NGF release from the central nervous system.

A problem in the current study may concern the observed trend toward a significant relationship between NGF values, and clinical and urodynamic results at baseline and during followup. These results did not attain statistical levels, probably because of the small study population. More interesting Spearman's correlation coefficient indicates that when NGF bladder tissue levels are increased, there are low UDC threshold and maximum bladder capacity values, and high UDC maximum pressure values. To our knowledge these correlations have not been documented previously. Even if these results are limited to postulate the reported mechanisms, they appear as logical consequence of the various effects of the neurotoxin. Further studies are needed to elucidate these effects.

\section{CONCLUSIONS}

In patients with SCI who have neurogenic detrusor overactivity refractory to standard anticholinergic treatment intravesical BTX-A injections provided beneficial clinical and urodynamic results with a decrease in detrusor overactivity and restoration of urinary incontinence. Furthermore, intravesical administration of the neurotoxin induced a state of NGF deprivation in bladder tissue that persisted up to 3 months after treatment. An explanation may be that the decrease in $\mathrm{ACh}$ release at the presynaptic level as the result of BTX-A induced decreases in detrusor contractility and NGF production. Another possibility is that BTX-A decreases neurogenic inflammation, thus, modulating bladder afferent nerve activity. A more intriguing mechanism is that BTX-A may decrease the level of neurotransmitters that normally modulate NGF production and release.

$\begin{aligned} & \text { Abbreviations and Acronyms } \\ \text { ACh }= & \text { acetylcholine } \\ \text { ATP } & =\text { adenosine triphosphate } \\ \text { BTX-A } & =\text { botulinum-A toxin } \\ \text { CGRP } & =\text { calcitonin gene-related peptide } \\ \text { NGF } & =\text { nerve growth factor } \\ \text { SCI } & =\text { spinal cord injury } \\ \text { UDC } & =\text { uninhibited detrusor contraction }\end{aligned}$

\section{REFERENCES}

1. Smith, C. P. and Chancellor, M. B.: Emerging role of botulinum toxin in the management of voiding dysfunction. J Urol, 171: 2128,2004

2. Cayan, S., Coskun, B., Bozlu, M., Acar, D., Akbay, E. and Ulusoy, E.: Botulinum toxin type A may improve bladder function in a rat chemical cystitis model. Urol Res, 30: 399, 2003

3. Duggan, M. J., Quinn, C. P., Chaddock, J. A., Purkiss, J. R., Alexander, F. C. G., Doward, S. et al: Inhibition of release of neurotransmitters from rat dorsal root ganglia by a novel conjugate of a clostridium botulinum toxin A endopeptidase fragment and erythrina cristagalli lectin. J Biol Chem, 277: 34846, 2002
4. de Groat, W. D., Kawatani, M., Hisamitsu, T., Cheng, C. L., Ma, C. P., Thor, K. et al: Mechanisms underlying the recovery of urinary bladder function following spinal cord injury. J Auton Nerv Syst, suppl., 30: 371, 1990

5. Steers, W. D., Kolbeck, S., Creedon, D. and Tuttle, J. B.: Nerve growth factor in the urinary bladder of the adult regulates neuronal form and function. J Clin Invest, 88: 1709, 1991

6. Ditunno, J. F., Jr., Young, W., Donovan, W. H. and Creasey, G.: The international standards booklet for neurological and functional classification of spinal cord injury. American Spinal Injury Association. Paraplegia, 32: 70, 1994

7. Stohrer, M., Goepel, M., Kondo, A., Kramer, G., Madersbacher, H., Millard, R. et al: The standardization of terminology in neurogenic lower urinary tract dysfunction: with suggestions for diagnostic procedures. International Continence Society Standardization Committee. Neurourol Urodyn, 18: 139, 1999

8. Okragly, A. J. and HaakFrendscho, M.: An acid-treatment method for the enhanced detection of GDNF in biological samples. Exp Neurol, 145: 592, 1997

9. Smith, C. P., Fraser, M. O, Bartho, L., deGroat, W. C., Chancellor, M. B. and Somogyi, G. T.: Botulinum toxin A inhibits afferent nerve evoked bladder strip contractions. J Urol, suppl., 167: 41, abstract 164, 2002

10. Cruz, F.: Mechanisms involved in new therapies for overactive bladder. Urology, 63: 65, 2004

11. Tanner, R., Chambers, P., Khadra, M. H. and Gillespie, J. I.: The production of nerve growth factor by human bladder smooth muscle cells in vivo and in vitro. BJU Int, 85: 1115, 2000

12. Lowe, E. M., Anand, P., Terenghi, G., Williams-Chestnut, R. E., Sinicropi, D. V. and Osborne, J. L.: Increased nerve growth factor levels in the urinary bladder of women with idiopathic sensory urgency and interstitial cystitis. $\mathrm{Br} \mathrm{J}$ Urol, 79: 572, 1997

13. Giannantoni, A., Di Stasi, S. M., Nardicchi, V., Macchioni, L., Guercini, F., Goracci, G. et al: Botulinum A toxin intravesical treatment induces a reduction of nerve growth factor bladder tissue levels in patients with neurogenic detrusor overactivity. J Urol, suppl., 173: 330, abstract 1217, 2005

14. Steers, W.D., Creedon, D. J. and Tuttle, J. B.: Immunity to nerve growth factor prevents afferent plasticity following urinary bladder hypertrophy. J Urol, 155: 379, 1996

15. Coderre, T. C., Basbaum, A. I. and Levine, J. D.: Neural control of vascular permeability: interactions between primary afferents, mast cells, and sympathetic efferents. J Neurophysiol, 62: 48, 1989

16. Smith, C. P., Franks, M. E., McNeil, B. K., Ghosh, R., de Groat W. C., Chancellor, M. B. et al: Effect of botulinum toxin A on the autonomic nervous system of the lower urinary tract. J Urol, 169: 1896, 2003

17. Khera, M., Somogyi, G. T., Kiss, S., Boone, T. B. and Smith, C. P.: Botulinum A toxin inhibits ATP release from bladder urothelium after chronic spinal cord injury. Neurochem Int, 45: 987, 2004

18. Chaddock, J. A., Purkiss, J. R., Duggan, M. J., Quinn, C. P., Shone, C. C. and Foster, K. A.: A conjugate composed of nerve growth factor coupled to a non-toxic derivative of clostridium botulinum neurotoxin type A can inhibit neurotransmitter release in vitro. Growth Factors, 18: 147, 2000

19. Seki, S., Sasaki, K., Fraser, M. O., Igawa, Y., Nishizawa, O., Chancellor, M. B. et al: Immunoneutralization of nerve growth factor in lumbosacral spinal cord reduces bladder hyperreflexia in spinal cord injured rats. J Urol, 168: 2269, 2002 